\title{
ON MAXIMAL FUNCTION AND FRACTIONAL INTEGRAL, ASSOCIATED WITH THE BESSEL DIFFERENTIAL OPERATOR
}

\author{
VAGIF S. GULIEV
}

Abstract. In this paper we consider the generalized shift operator, generated by Bessel differential operator $B$, by means of which maximal functions ( $B$-maximal functions) and fractional integrals ( $B$-fractional integrals) are investigated. The $L_{p}(B)$-boundedness result for the $B$-maximal function and $\left(L_{p}(B), L_{q}(B)\right)$-boundedness result for the $B$-fractional integral are obtained.

Mathematics subject classification (2000): 47F05, 46E35.

Key words and phrases: Bessel differential operator, $B$-maximal function, $B$-fractional integral.

\section{REFERENCES}

[1] B. M. LEVITAN, Bessel function expansions in series and Fourier integrals. Uspekhi Mat. Nauk 42(2) (1951), 102-143.

[2] V. S. Guliev, Sobolev theorems for B-Riesz potentials. Dokl. RAN, 358(4) (1998), p. 450-451.

[3] V. S. GuLIEV, Sobolev theorems for anisotropic Riesz-Bessel potentials on Morrey-Bessel spaces. Dokl. RAN, 367(2) (1999), p. 155-156.

[4] R. R. CoIfman, G. Weiss, Analyse harmonique non commutative sur certains expaces homogenes. Lecture Notes in Math., v. 242, Springer-Verlag. Berlin, 1971.

[5] K. SteMPaK, Almost everywhere summability of Laguerre series, Studia Math. 100(2) (1991), 129-147. 